\title{
Research Paper \\ Comparison of Balance and Strength of Lower Limb Muscles Between two Groups of People With Covid-19 and Healthy Ones: A Cross-Sectional Study
}

\section{${ }^{*}$ Amir Ali Jafarnezhadgero ${ }^{1}$ (I), Amin Hoseinpour ${ }^{1}$}

1. Department of Sports Management and Biomechanics, Faculty of Education Sciences and Psychology, University of Mohaghegh Ardabili, Ardabil, Iran

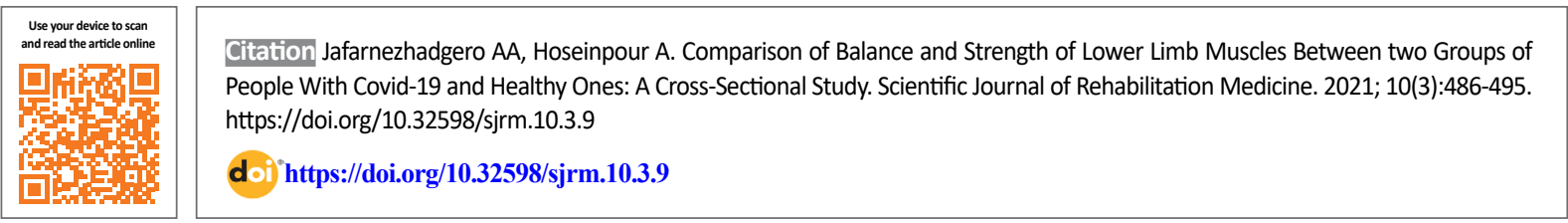

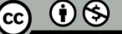

Received: 12 Mar 2021 Accepted: ?30 Apr 2021? Available Online: 23 Jul 2021

Keywords:

Postural balance, Lower extremity, Covid-19

\section{A B STR ACT}

Background and Aims This study evaluated dynamic balance, quadriceps, and hamstring strength in individuals with Covid 19 compared with healthy control ones.

Methods A total of 30 people were divided into two groups, including healthy people $(n=15)$ and people with Covid-19. Dynamic balance was measured using the star excursion balance test. A dynamometer performed lower limb muscle strength measurements. An independent t-test was used to compare the two groups statistically. The significance level was considered 0.05 .

Results Quadriceps and hamstring muscle strength were similar in both groups $(P>0.05)$. The results showed that the values of dynamic balance in the lateral $(P=0.001)$, medial $(P=0.001)$, and posterior medial $(P=0.001)$ directions were significantly lower in the group with Covid-19 than that in the healthy group. Conclusion In general, the balance of people with Covid-19 was significantly lower than the healthy group. Decreased balance due to this disease can increase the risk of injury. There was no significant difference in lower limb muscle strength in patients with Covid-19 compared with healthy individuals. Perhaps more research is needed in the future to prove this better.

\section{Extended Abstract}

\section{Introduction}

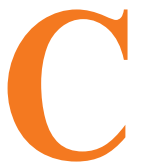

oronavirus Disease (COVID-19) is an infectious disease caused by a newly discovered coronavirus. Most people who fall sick with COVID-19 will experience mild to moderate symptoms and recover without special treatment. Dynamic balance is the ability to maintain postural stability and orientation with the center of mass over the support base while the body parts are in motion. The Star Excursion Balance Test (SEBT) is a dynamic test that requires strength, flexibility, and proprioception. It is a measure of dynamic balance that provides a significant challenge to athletes and physically active individuals. The test can be used to assess physical performance. Still, it can be used to screen deficits in dynamic postural control due to musculoskeletal injuries (e.g. chronic ankle instability), identify athletes at greater risk for lower extremity injury, and rehabilitate orthopedic injuries in healthy, active adults. Normative muscle strength data for defined populations of athletes are essential to sports coaches, athletic trainer, medicine physicians, physical therapists, and others responsible for athletes' health and specifically for return-to-play criteria. This study aimed to evaluate dynamic balance, quadriceps, and hamstring strength in individuals with Covid-19 compared with healthy control ones.

Corresponding Author:

Amir Ali Jafarnezhadgero, PhD.

Address: Department of Sports Management and Biomechanics, Faculty of Education Sciences and Psychology, University of Mohaghegh Ardabili, Ardabil, Iran.

E-Mail: amiralijafarnezhad@gmail.com 


\section{Methods}

A total of 30 people were divided into two groups, including healthy people $(n=15)$ and people with Covid-19. Dynamic balance was measured using the star excursion balance test. The person performing the test must maintain balance on one leg while using the other leg to reach as far as possible in 8 different directions. The person (standing on his/her left leg, for example) must run in 8 different positions, once in each of the following directions: anterior, anteromedial, medial, posteromedial, posterior, posterolateral, lateral, and anterolateral. A dynamometer performed lower limb muscle strength measurements. Shapiro-Wilk test confirmed the normal distribution of data. An independent sample t-test was used to compare the two groups statistically. The significance level was considered 0.05 . All analyses were done by SPSS Software v. 22

\section{Results}

Quadriceps and hamstring muscle strength were similar in both groups $(\mathrm{P}>0.05)$. The results showed that the values of dynamic balance in the lateral $(\mathrm{P}=0.001)$, medial $(\mathrm{P}=0.001)$, and posterior medial $(\mathrm{P}=0.001)$ directions were significantly lower in the group with Covid-19 than that in the healthy group.

\section{Discussion and Conclusion}

In general, the balance of people with Covid-19 was significantly lower than the healthy group. Decreased balance due to this disease can increase the risk of injury. An individual's quadriceps and hamstring muscle strength is an important part of the athlete's functional capacity and contributes significantly to lower limb biomechanics and performance. There was no significant difference in lower limb muscle strength in patients with Covid-19 compared with healthy individuals. Perhaps more research is needed in the future to better prove this.

\section{Ethical Considerations}

\section{Compliance with ethical guidelines}

All ethical principles are considered in this article. The ethical principles observed in the article, such as the informed consent of the participants, the confidentiality of information, the permission of the participants to cancel their participation in the research.

\section{Funding}

This study is extracted from the research project of the first author in the Department of Sports Management and Biomechanics of Mohaghegh Ardabili University.

\section{Authors' contributions}

Authors contributed equally in preparing this article.

\section{Conflict of interest}

The authors declared no conflict of interest. 
This Page Intentionally Left Blank 
مقاله يخؤوهشى

مقايسه تعادل و قدرت عضالات اندام تحتانى بين دو تروه افراد مبتلا به كوويد-19 و افراد ساله: يكى مطالعه عرضى

\author{
"اميرعلى جعفرنزادكرو' هـ، امين حسينيور'
}

ا. كروه مديريت و بيومكانيك ورزشى، دانشكده علوم تربيتى و روانشناسى، دانشكاه محقق اردبيلى، اردبيل، ايران.

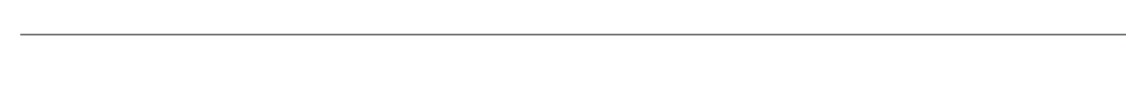

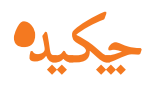

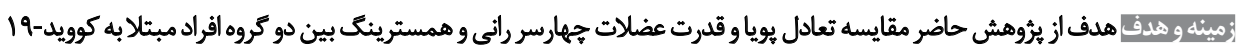
و همسالان سالم بود.

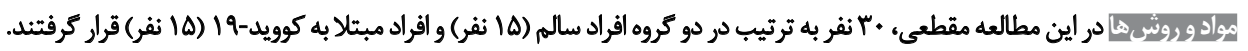

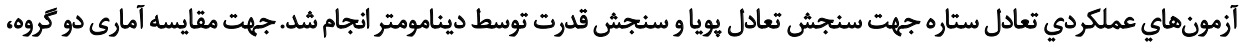

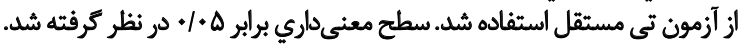

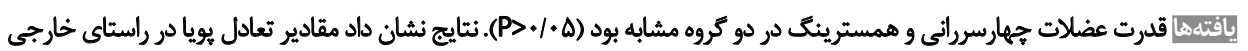

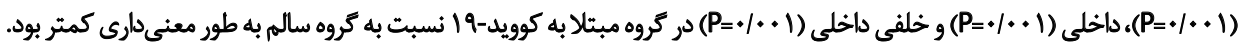

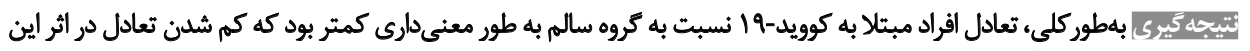

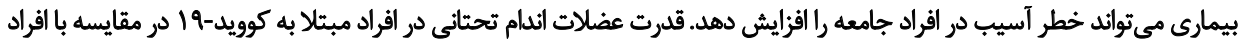

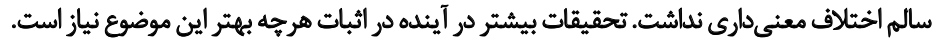

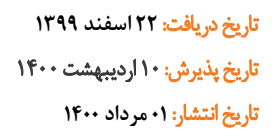

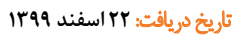

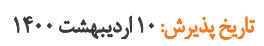

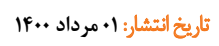

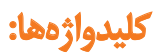

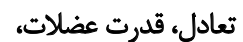
الندام تحتاني، كوويد-19 ت ت

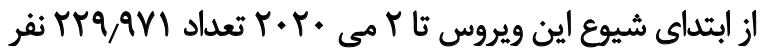

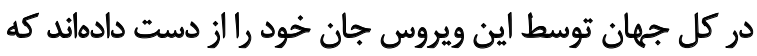

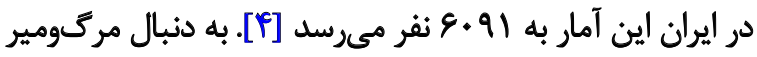

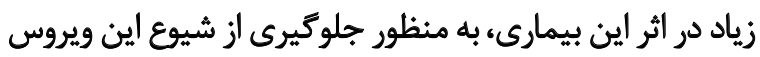

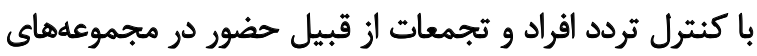

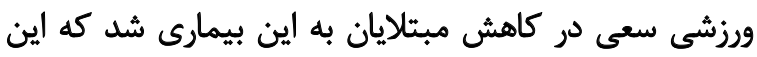

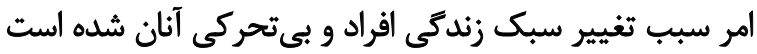

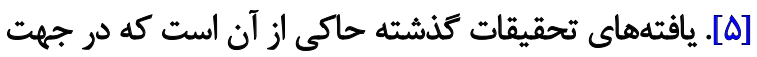

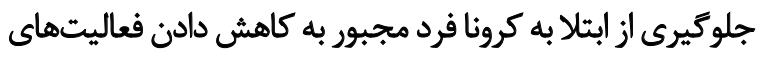

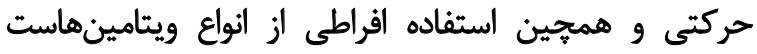

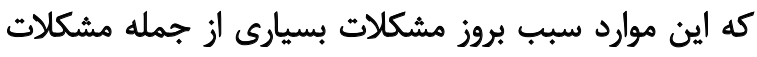

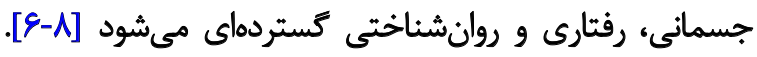

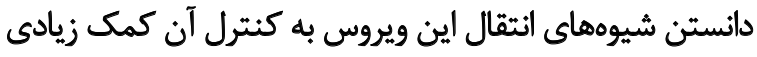

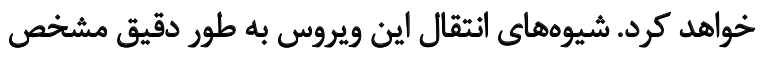

كروناويروس در سال 19 •r (Covid-19) براى اولينبار در

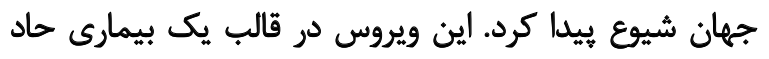

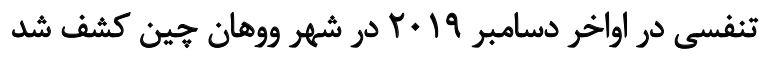

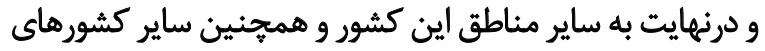

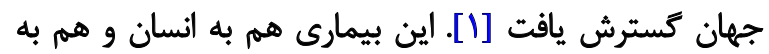

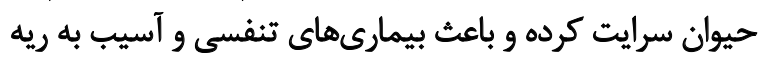

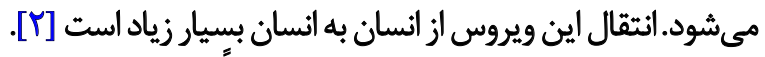
علامت اوليه اين ويروس ذات الريه است و اخيراً به اختلال دستئاه

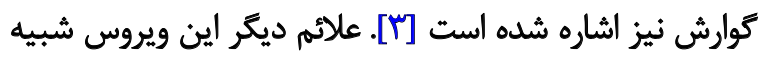

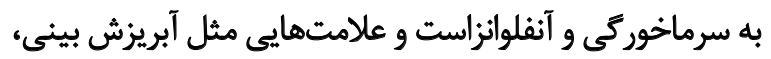

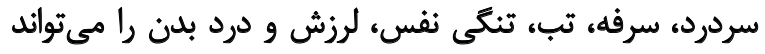

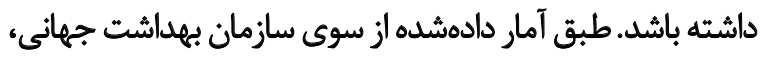

$$
\begin{aligned}
& \text { "نويسنده مسئول: }
\end{aligned}
$$

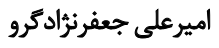

$$
\begin{aligned}
& \text { نشائي: اردبيل، دائشكاه محقق اردبيلي، دانشكده علوم تربيتى و روانشناسي، كروه مديريت و بيومكانيك ورزشي. }
\end{aligned}
$$

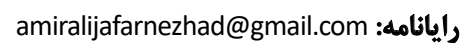


سقوطهاى متوالى باعث شكستكى در اندام يا حتى سبب مرتى

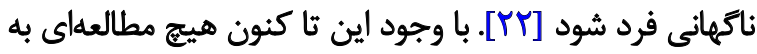

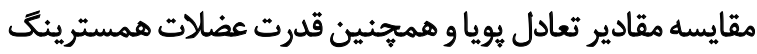

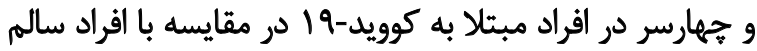

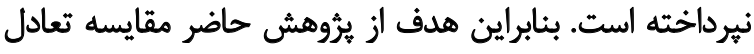

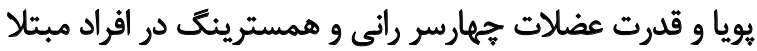

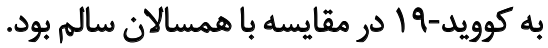

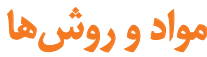

يُروهش حاضر از نوع مقطعى و عرضى است. جامعه آمارى

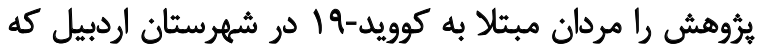

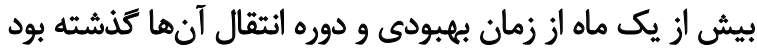

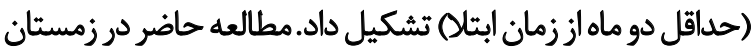

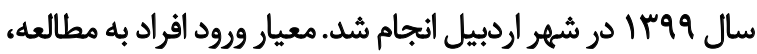

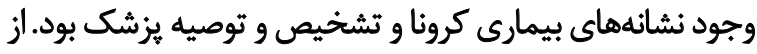

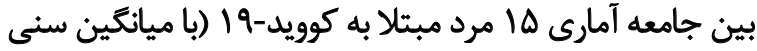

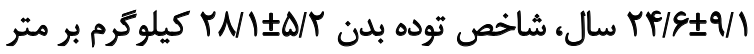

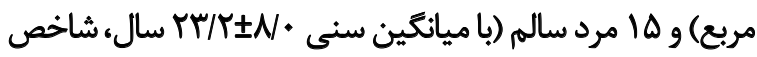
توده بدن

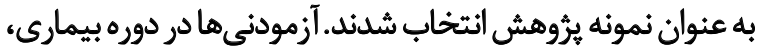

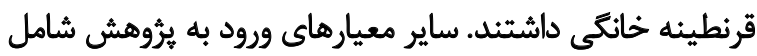

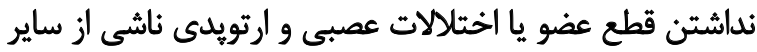

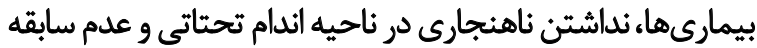

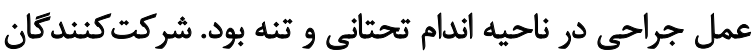

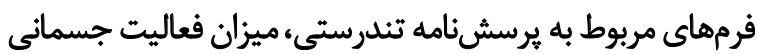

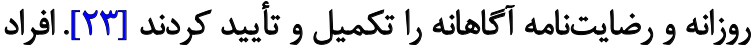

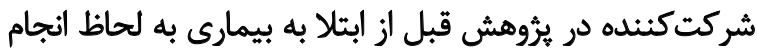
فعاليت فيزيكى منظم، غيرفعال بودند.

بيش از شروع آزمون، تمام نكات بهداشتى از جمله استفاده از

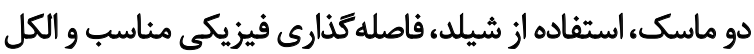

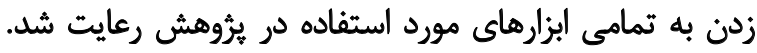

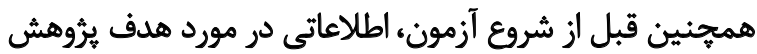

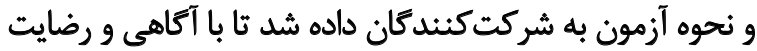

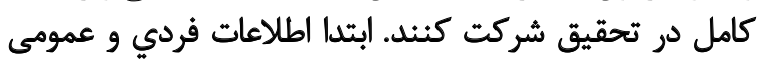

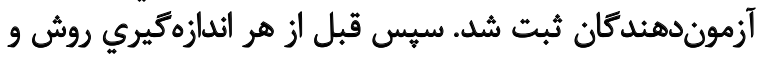

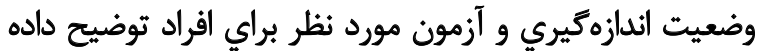

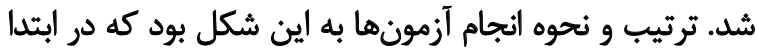

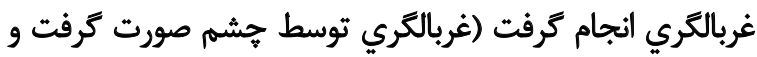

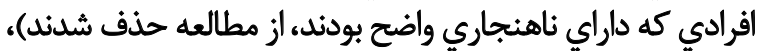

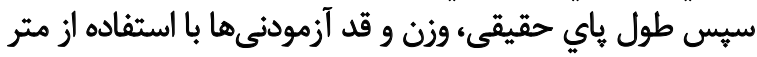

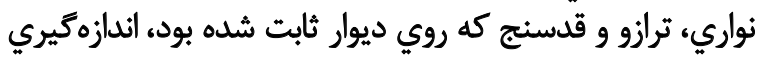
شد. براي تعيين ياي برتر از روشهاي زئ زير وير استفاده شد:

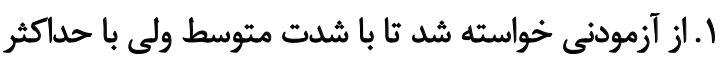

نيست، ولى به صورت كلى مىتوان كفت ويروسهاى تنفسى از

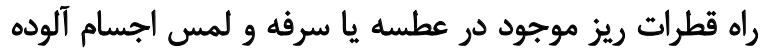

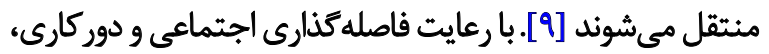

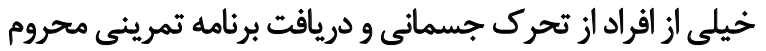

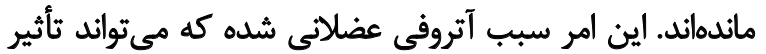

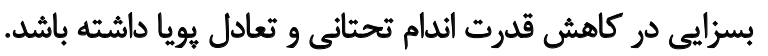

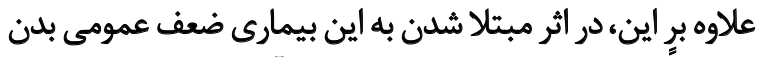

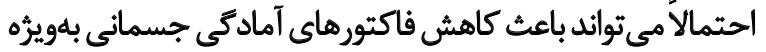

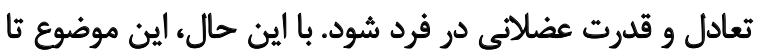

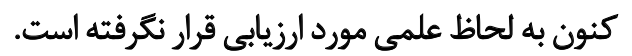

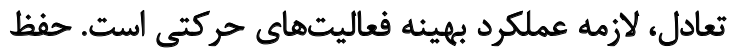

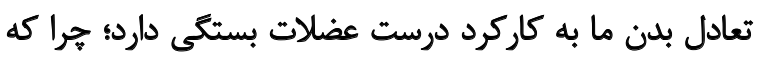

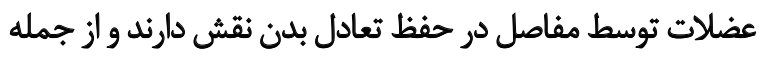

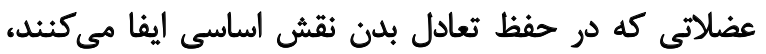

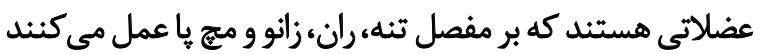

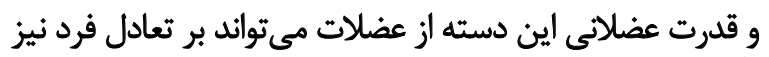

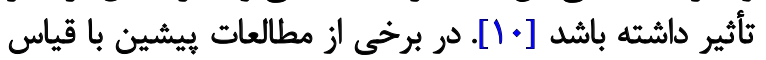

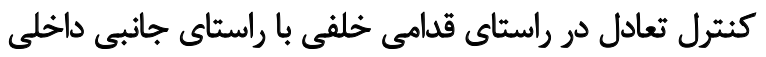

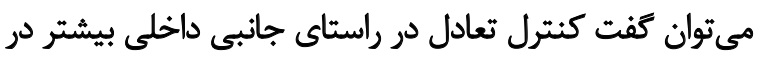

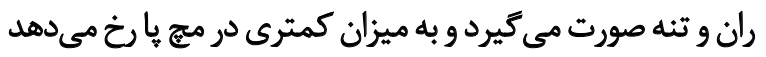

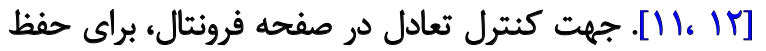

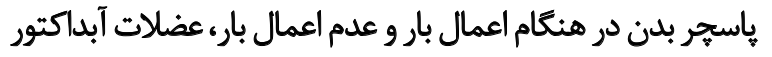

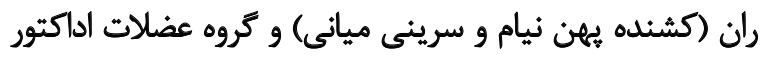

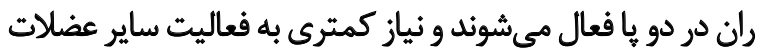

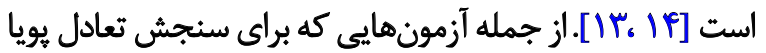

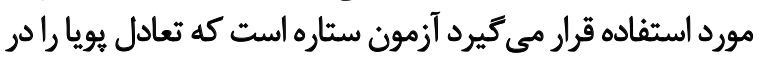

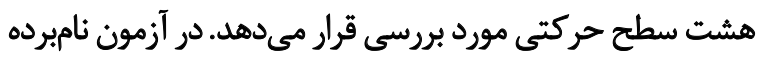

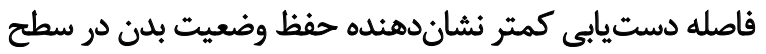

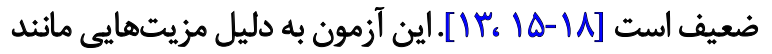

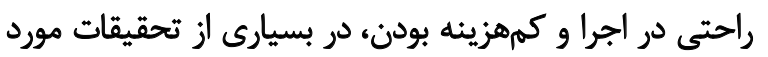

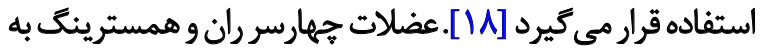

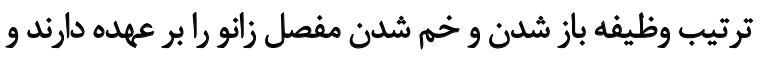

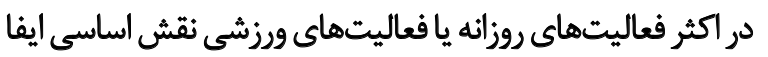

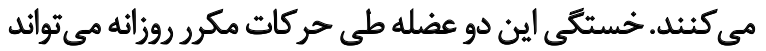

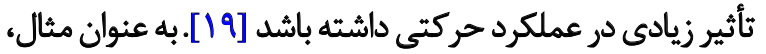

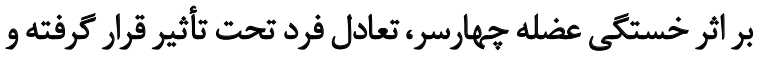

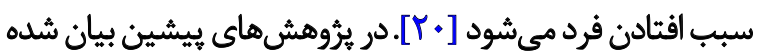

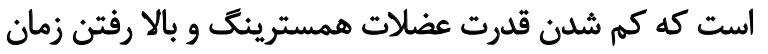

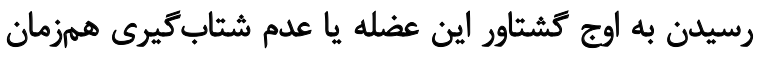

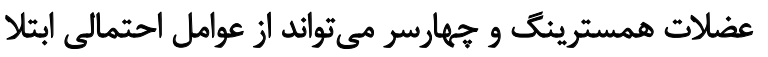

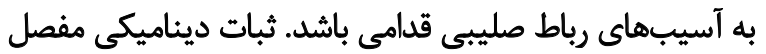

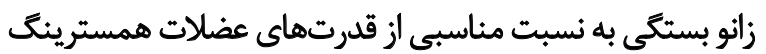

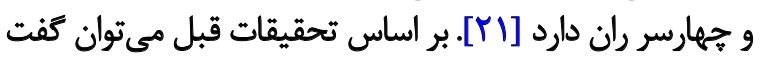

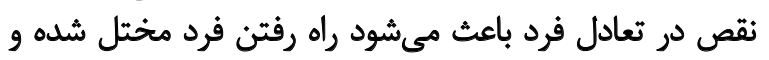


بافتهنا

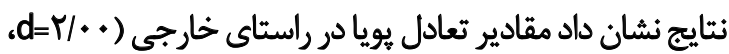

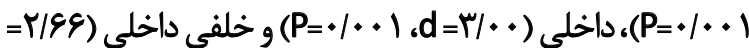

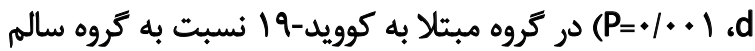

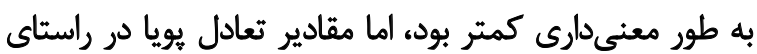

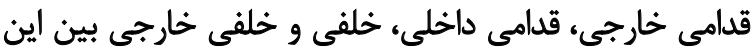

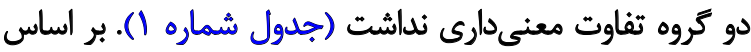

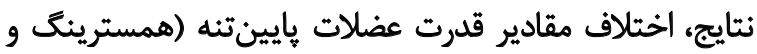

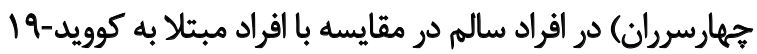

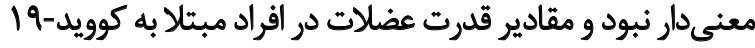

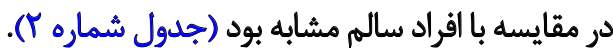

ث

هدف از انجام اين تحقيق مقايسه قدرت عضلات همسترينك،

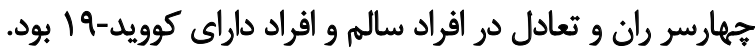

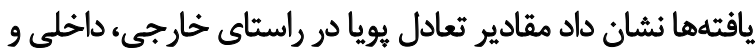

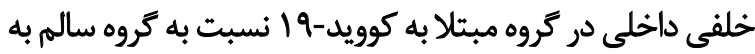

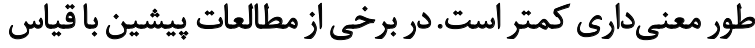

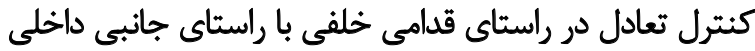

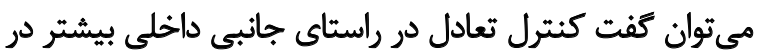

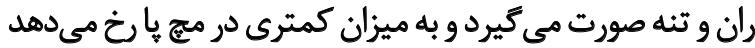

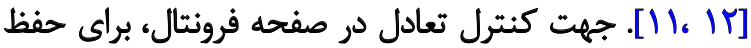

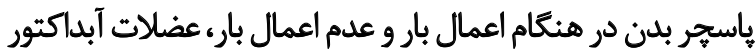

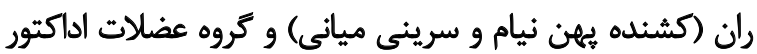

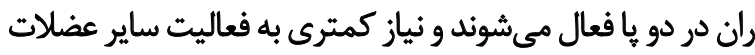

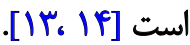

يافتهها نشان داد اختلاف مقادير تعادل يويا در راستاى قدامى

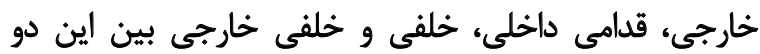

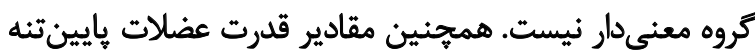

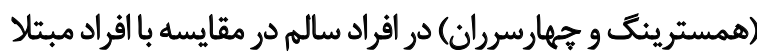

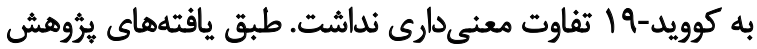

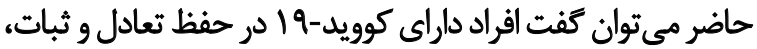

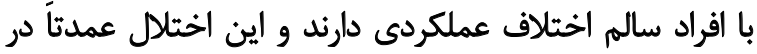



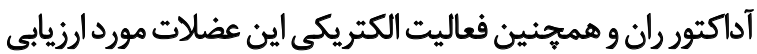

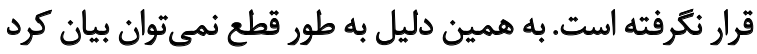

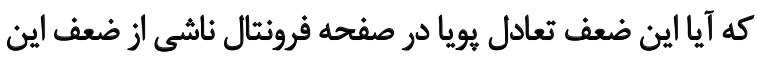

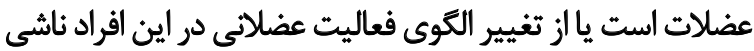
مىشود. بنابراين مطالعات بيشتر در اين زمينه توصيه مى فئردا

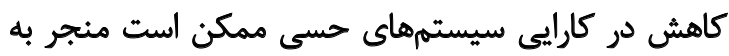

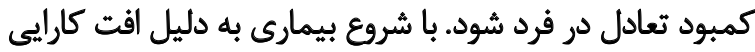
سيستم حسى بينايى، وستيبولار و حسى عمقى احتى احتمال اختلال
دقت توب فوتبال را به سمت دروازهاي به طول يك متر كه در

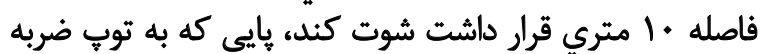

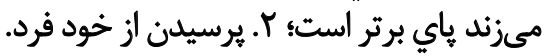

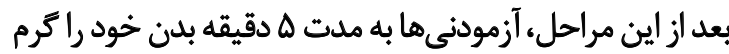

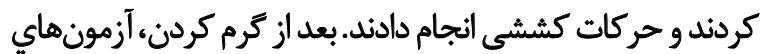

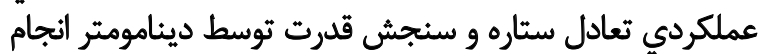

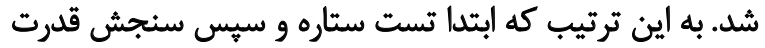

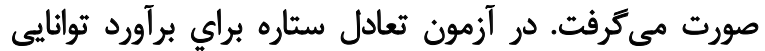

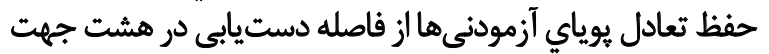

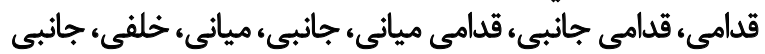

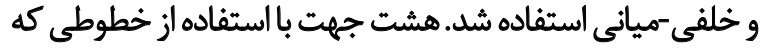

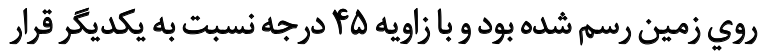

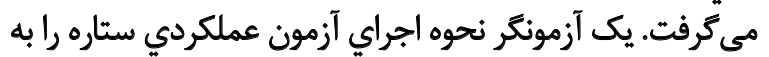

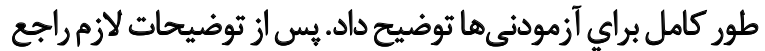

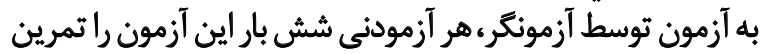

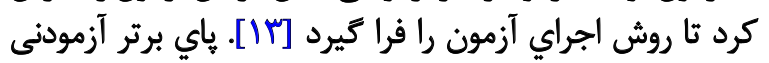

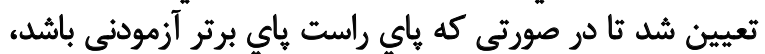

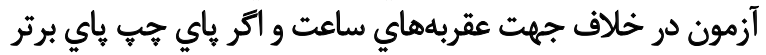

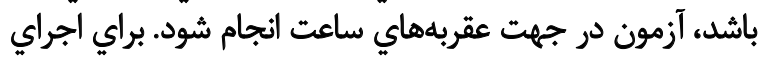

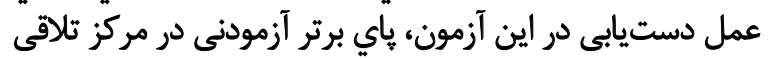

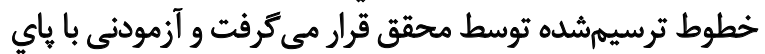

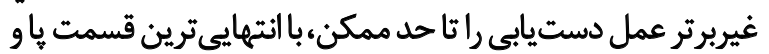

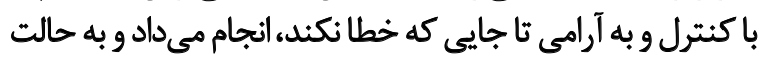

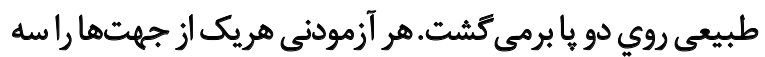

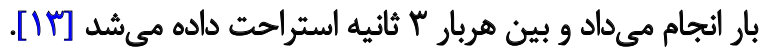

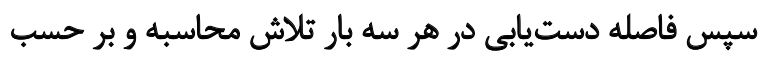

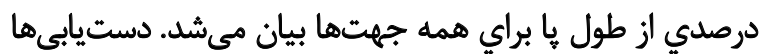

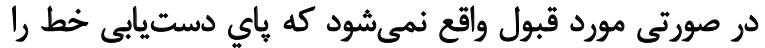

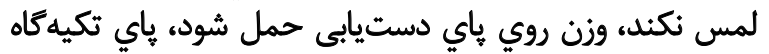

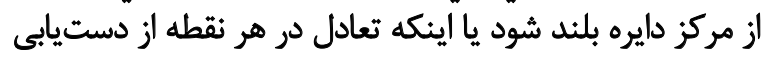

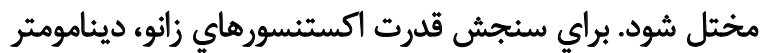

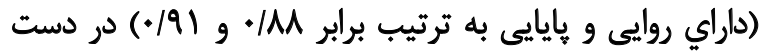

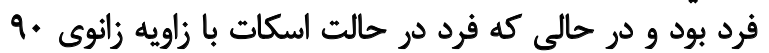

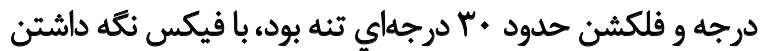

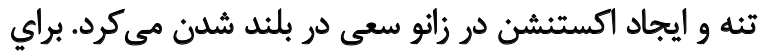

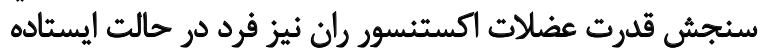

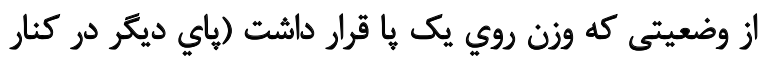

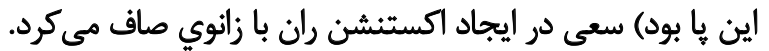

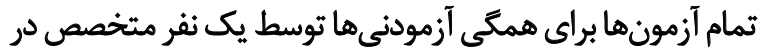

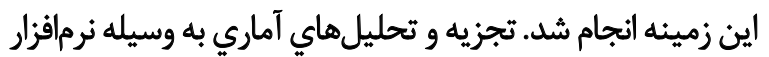

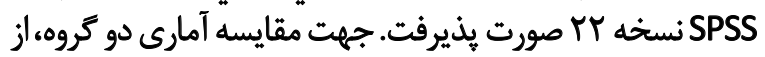
آزمون تى مستقل و جهت محاسبه مقادير اندازه اثثر از رابطه كوهنز

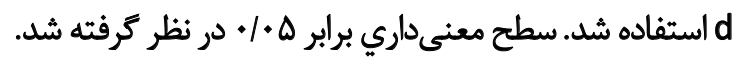


جدول ا. مقايسه مقادير تعادل يويا در افراد مبتلا به كوويد-9 أدر مقايسه با افراد سالم

\begin{tabular}{|c|c|c|c|c|c|}
\hline \multirow{2}{*}{ اندازه اثر } & \multirow{2}{*}{ سطح معنىدارى } & \multirow{2}{*}{$\mathbf{t}$} & \multicolumn{2}{|c|}{ مياتكين=|نحرافقمعيار } & \multirow{2}{*}{ تعادل } \\
\hline & & & تروه كوويد-19 & تروه سالم & \\
\hline.$/ T a$ &.$/ N T$ & $-V / \Delta H$ & $A * / Q T \pm V / T 1$ & 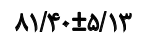 & قدامى خارجى \\
\hline.$/ T \Delta$ & $\cdot / \Delta \cdot V$ & $\lg \pi$ & $W / F E \pm \Delta / T Y$ & WNE. \pm Y/QY & قدامى داخلى \\
\hline$r / .$. & $.1 . .1$ & $-r / \pi V \lambda$ & $E \cdot R E \pm F / R$ & $\Delta r / F E \pm T / \Delta F$ & خارجى \\
\hline$r \%$ & $.1 . .1$ & $-ه / \& \& 9$ & AT/TE \pm TAF & $V \& / q T \pm Y / T A$ & داخلى \\
\hline .198 & $\cdot / r+\Delta$ & $-1 /$ rqv & $9 r / 4 \& \pm r / 19$ & $q . / N E \pm F / T$ & خلفى \\
\hline.$/ r$ & $\cdot / f+r$ & ./NA. & $9+/ 1 r \pm \Delta / 18$ & $9) / N E \pm$ / 9 V & خلفى خارجى \\
\hline T/E & $.1 . .1$ & $-Q / 9 V r$ & $9.1 .9 \pm 1 / 79$ & $V N T T \pm P / A)$ & خلفى داخلى \\
\hline
\end{tabular}

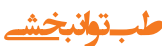

كنترل كننده حركات اندام تحتانى در صفحه فرونتال در افروان افراد

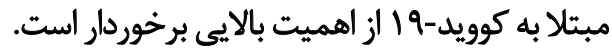

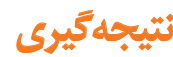

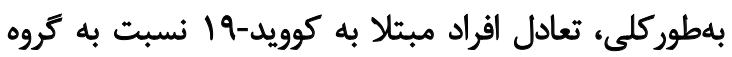

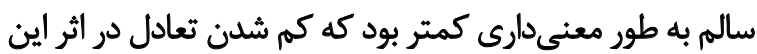

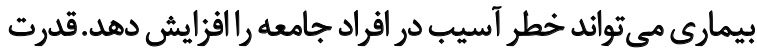

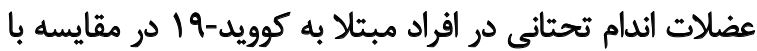

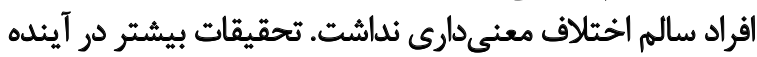

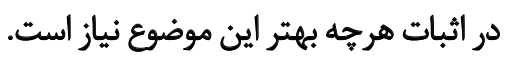

ئروهش حاضر داراى محدوديتهايى بود كه از آن جمله

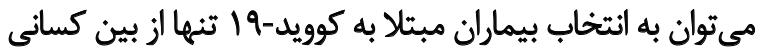

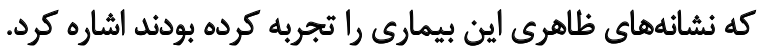

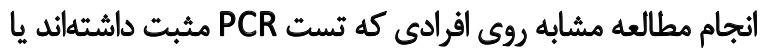

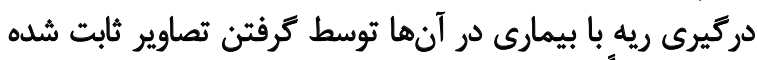

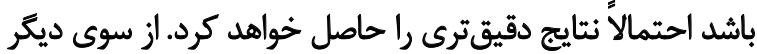

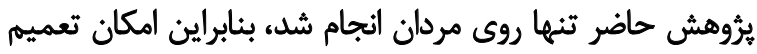

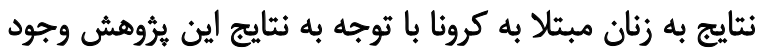

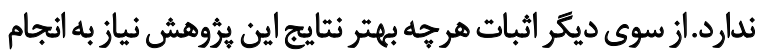

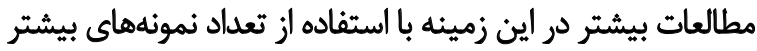

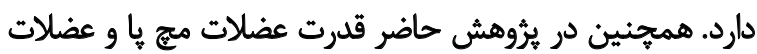

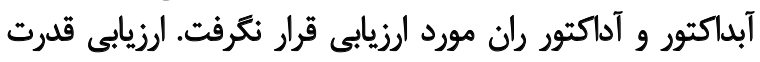

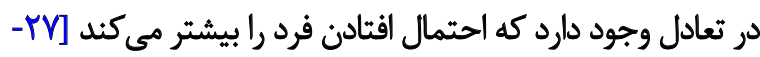

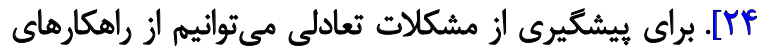

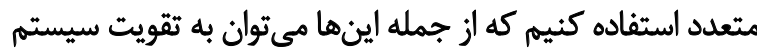

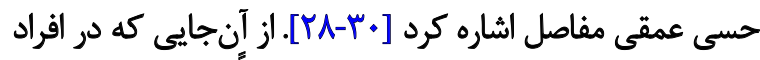

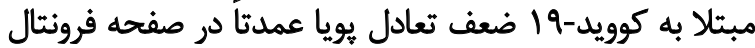

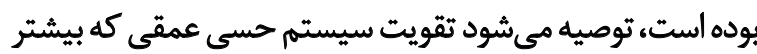

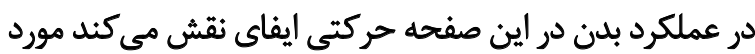

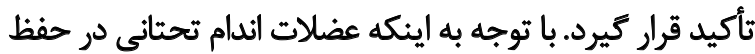



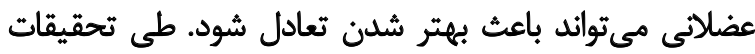

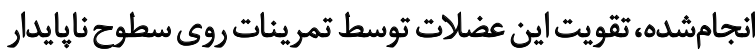

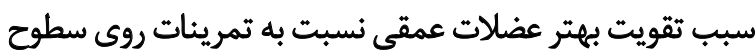

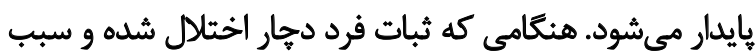

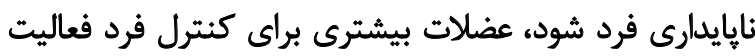

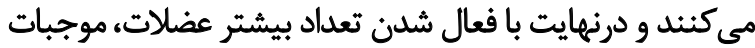

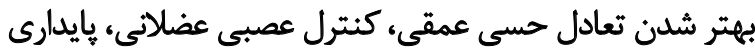

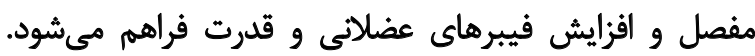

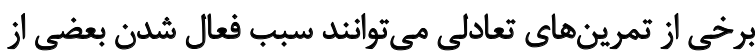

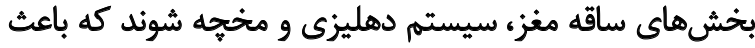

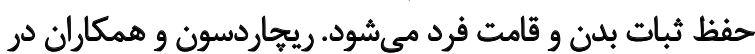

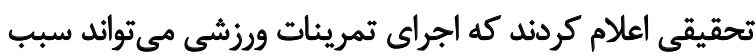

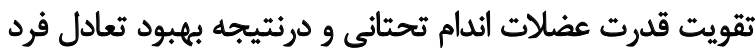

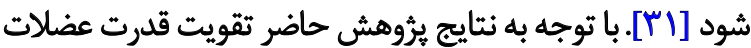

جدول r. مقايسه مقادير قدرت عضلات در افراد مبتلا به كوويد-19 در مقايسه با افراد سالم

\begin{tabular}{|c|c|c|c|c|c|}
\hline \multirow{2}{*}{ اثدازه اثر } & \multirow{2}{*}{ سطح معنى دارى } & \multirow{2}{*}{$\mathbf{t}$} & \multicolumn{2}{|c|}{ ميانكين士|نحرافمعيار } & \multirow{2}{*}{ قدرت عضلات } \\
\hline & & & كروه كوويد-19 & كروه سالم & \\
\hline$. / \Delta<\Delta$ &.$/ M T$ & $1 / 49$ & १Q/YระQ/10 & $1+4 / A \cdot \pm 9 / 79$ & جهارسرران \\
\hline - /NFa & . leqr & $-r q u$ & Vq/qu $\pm N A Y$ & $V N Y V^{\prime} \pm V / \Delta Q$ & همسترينى \\
\hline
\end{tabular}


اين عضلات مىتوائد اطلاعات جامعى را در اين زمينه در اختيار

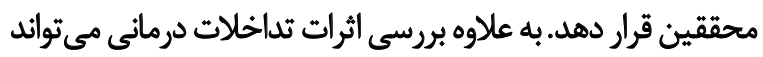

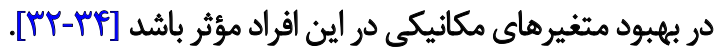
مالاحظاث الخالاقي يبروى ائز اصول اخلاق هُؤشش اصول اخلاقى تماماً در اين مقاله رعايت شده است. شركت

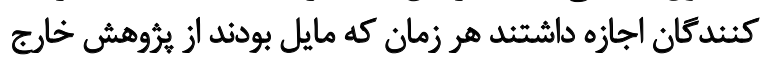

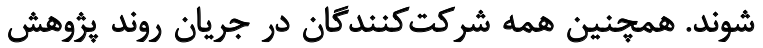

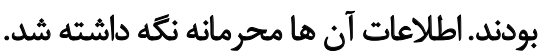

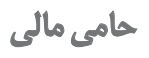

اين مطالعه از طرح ثروهشى نويسنده اول در تروه مديريت و

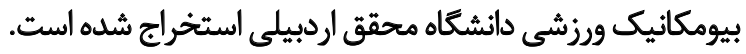

$$
\text { مشاركت نويسندكان }
$$

تمام نويسندكان در طراحى، اجرا و نتارش همه بخشهاى يُروهش حاضر مشاركت داشتهاند.

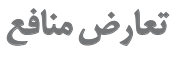

بنابر اظهار نويسندكان اين مقاله تعارض منافع ندارد. 


\section{References}

[1] Paules $\mathrm{Cl}$, Marston HD, Fauci AS. Coronavirus infectionsmore than just the common cold. JAMA. 2020; 323(8):707-8. [DOI:10.1001/jama.2020.0757] [PMID]

[2] Phillipou A, Meyer D, Neill E, Tan EJ, Toh WL, Van Rheenen TE, et al. Eating and exercise behaviors in eating disorders and the general population during the COVID-19 pandemic in Australia: Initial results from the COLLATE project. International Journal of Eating Disorders. 2020; 53(7):1158-65. [DOI:10.1002/ eat.23317] [PMID] [PMCID]

[3] Chan JF-W, Yuan S, Kok K-H, To KK-W, Chu H, Yang J, et al. A familial cluster of pneumonia associated with the 2019 novel coronavirus indicating person-to-person transmission: A study of a family cluster. The Lancet. 2020; 395(10223):514-23. [DOI:10.1016/S0140-6736(20)30154-9]

[4] Tavakoli A, Vahdat K, Keshavarz M. [Novel coronavirus disease 2019 (COVID-19): An emerging infectious disease in the 21st century (Persian)]. Iranian South Medical Journal. 2020; 22(6):432-50. [DOI:10.29252/ismj.22.6.432]

[5] Godman B. Combating COVID-19: Lessons learnt particularly among developing countries and the implications. Bangladesh Journal of Medical Science. 2020; 19(Special Issue on Covid19):S103-8. [DOI:10.3329/bjms.v19i0.48413]

[6] Fernández-Aranda F, Casas M, Claes L, Bryan DC, Favaro A, Granero R, et al. COVID-19 and implications for eating disorders. European Eating Disorders Review. 2020; 28(3):239. [DOI:10.1002/erv.2738] [PMID] [PMCID]

[7] Weissman RS, Bauer S, Thomas JJ. Access to evidence-based care for eating disorders during the COVID-19 crisis. International Journal of Eating Disorders. 2020; 53(5):369-76. [DOI:10.1002/eat.23279] [PMID] [PMCID]

[8] Martinez-Ferran M, de la Guía-Galipienso F, Sanchis-Gomar F, Pareja-Galeano H. Metabolic impacts of confinement during the COVID-19 pandemic due to modified diet and physical activity habits. Nutrients. 2020; 12(6):1549. [DOI:10.3390/ nu12061549] [PMID] [PMCID]

[9] WHO. Clinical management of severe acute respiratory infection when novel coronavirus (2019-nCoV) infection is suspected: Interim guidance. Geneva: World Health Organization; 2020. https://apps.who.int/iris/handle/10665/330893

[10] Lyytinen T, Liikavainio T, Bragge T, Hakkarainen M, Karjalainen PA, Arokoski JP. Postural control and thigh muscle activity in men with knee osteoarthritis. Journal of Electromyography and Kinesiology. 2010; 20(6):1066-74. [DOI:10.1016/j.jelekin.2010.05.005] [PMID]

[11] Day B, Steiger M, Thompson P, Marsden C. Effect of vision and stance width on human body motion when standing: Implications for afferent control of lateral sway. The Journal of Physiology. 1993; 469:479-99. [DOI:10.1113/jphysiol.1993.sp019824] [PMID] [PMCID]

[12] Kapteyn T. Afterthought about the physics and mechanics of the postural sway. Agressologie. 1973; 14(Spec No C):27-35. [PMID]

[13] Hertel J, Braham RA, Hale SA, Olmsted-Kramer LC. Simplifying the star excursion balance test: Analyses of subjects with and without chronic ankle instability. Journal of Orthopaedic \& Sports Physical Therapy. 2006; 36(3):131-7. [DOI:10.2519/ jospt.2006.36.3.131] [PMID]

[14] Winter DA, Prince F, Stergiou P, Powell C. Medial-lateral and anterior-posterior motor responses associated with center of pressure changes in quiet standing. Neuroscience Research Communications. 1993; 12(3):141-8. https://eurekamag.com/ research/009/005/009005811.php

[15] Olmsted LC, Carcia CR, Hertel J, Shultz SJ. Efficacy of the star excursion balance tests in detecting reach deficits in subjects with chronic ankle instability. Journal of Athletic Training. 2002; 37(4):501-6. [PMCID]

[16] Gribble PA, Hertel J. Considerations for normalizing measures of the Star Excursion Balance Test. Measurement in Physical Education and Exercise Science. 2003; 7(2):89-100. [DOI:10.1207/ S15327841MPEE0702_3]

[17] Reiman MP, Manske RC. Functional testing in human performance. Champaign: Human kinetics; 2009. [DOI:10.5040/9781492596882]

[18] Hoch MC, Staton GS, McKeon JMM, Mattacola CG, McKeon PO. Dorsiflexion and dynamic postural control deficits are present in those with chronic ankle instability. Journal of Science and Medicine in Sport. 2012; 15(6):574-9. [DOI:10.1016/j. jsams.2012.02.009] [PMID]

[19] Paillard T. Effects of general and local fatigue on postural control: A review. Neuroscience \& Biobehavioral Reviews. 2012; 36(1):162-76. [DOI:10.1016/j.neubiorev.2011.05.009] [PMID]

[20] Hassani A, Patikas D, Bassa E, Hatzikotoulas K, Kellis E, Kotzamanidis $\mathrm{C}$. Agonist and antagonist muscle activation during maximal and submaximal isokinetic fatigue tests of the knee extensors. Journal of Electromyography and Kinesiology. 2006; 16(6):661-8. [DOI:10.1016/j.jelekin.2005.11.006] [PMID]

[21] Hortobágyi T, Westerkamp L, Beam S, Moody J, Garry J, Holbert $D$, et al. Altered hamstring-quadriceps muscle balance in patients with knee osteoarthritis. Clinical Biomechanics. 2005; 20(1):97104. [DOI:10.1016/j.clinbiomech.2004.08.004] [PMID]

[22] Gadelha AB, Neri SGR, Oliveira RJd, Bottaro M, David ACd, Vainshelboim $B$, et al. Severity of sarcopenia is associated with postural balance and risk of falls in community-dwelling older women. Experimental Aging Research. 2018; 44(3):258-69. [DOI:10.1080/ 0361073X.2018.1449591] [PMID]

[23] American College of Sports Medicine. ACSM's health-related physical fitness assessment manual. Philadelphia: Lippincott Williams \& Wilkins; 2013. https://books.google.com/ books/about/ACSM_s_Health_Related_Physical_Fitness_A. html?id=ZPo96rd3PpAC

[24] Horak FB, Nashner LM. Central programming of postural movements: Adaptation to altered support-surface configurations. Journal of Neurophysiology. 1986; 55(6):1369-81. [DOI:10.1152/ jn.1986.55.6.1369] [PMID]

[25] Brandt T, Dieterich M. Vestibular falls. Journal of Vestibular Research: Equilibrium and Orientation. 1993; 3(1):3-14. https:// www.safetylit.org/citations/index.php?fuseaction=citations.vi ewdetails\&citationlds[]=citjournalarticle_156932_30 
[26] Skinner HB, Barrack RL, Cook SD. Age-related decline in proprioception. Clinical Orthopaedics and Related Research. 1984; (184):208-11. [DOI:10.1097/00003086-19840400000035]

[27] Gardner MM, Buchner DM, Robertson MC, Campbell AJ. Practical implementation of an exercise-based falls prevention programme. Age and Ageing. 2001; 30(1):77-83. [DOI:10.1093/ageing/30.1.77] [PMID]

[28] Lephart SM, Pincivero DM, Giraido JL, Fu FH. The role of proprioception in the management and rehabilitation of athletic injuries. The American Journal of Sports Medicine. 1997; 25(1):130-7. [DOI:10.1177/036354659702500126] [PMID]

[29] Laskowski ER, Newcomer-Aney K, Smith J. Proprioception. Physical Medicine and Rehabilitation Clinics of North America. 2000; 11(2):323-40. [DOI:10.1016/S1047-9651(18)30132-3]

[30] Thomeé R, Augustsson J, Karlsson J. Patellofemoral pain syndrome: A review of current issues. Sports Medicine. 1999; 28(4):245-62. [DOI:10.2165/00007256-199928040-00003] [PMID]

[31] Richardson JK, Sandman D, Vela S. A focused exercise regimen improves clinical measures of balance in patients with peripheral neuropathy. Archives of Physical Medicine and Rehabilitation. 2001; 82(2):205-9. [DOI:10.1053/apmr.2001.19742] [PMID]

[32] Madadi-Shad M, Jafarnezhadgero AA, Sheikhalizade H, Dionisio VC. Effect of a corrective exercise program on gait kinetics and muscle activities in older adults with both low back pain and pronated feet: A double-blind, randomized controlled trial. Gait \& Posture. 2020; 76:339-45. [DOI:10.1016/j.gaitpost.2019.12.026] [PMID]

[33] Jafarnezhadgero AA, Anvari M, Granacher U. Long-term effects of shoe mileage on ground reaction forces and lower limb muscle activities during walking in individuals with genu varus. Clinical Biomechanics. 2020; 73:55-62. [DOI:10.1016/j. clinbiomech.2020.01.006] [PMID]

[34] Jafarnezhadgero A, Ghorbanloo F, Fatollahi A, Dionisio VC, Granacher U. Effects of an elastic resistance band exercise program on kinetics and muscle activities during walking in young adults with genu valgus: A double-blinded randomized controlled trial. Clinical Biomechanics. 2021; 81:105215. [DOI:10.1016/j.clinbiomech.2020.105215] [PMID] 